\title{
Oral Class I and III antiarrhythmic drugs for maintaining sinus rhythm after catheter ablation of atrial fibrillation (Protocol)
}

Warraich M, Peter C, Ahmad M, Sheikh S, Abraham GR, Sahibzada MS, Baral R, Muzaffar A, Tomson J, Lambiase P, Captur G, Banerjee A, Providencia R

Warraich M, Peter C, Ahmad M, Sheikh S, Abraham GR, Sahibzada MS, Baral R, Muzaffar A, Tomson J, Lambiase P, Captur G, Banerjee A, Providencia R.

Oral Class I and III antiarrhythmic drugs for maintaining sinus rhythm after catheter ablation of atrial fibrillation (Protocol).

Cochrane Database of Systematic Reviews 2020, Issue 10. Art. No.: CD013765.

DOI: 10.1002/14651858.CD013765.

www.cochranelibrary.com

Oral Class I and III antiarrhythmic drugs for maintaining sinus rhythm after catheter ablation of atrial fibrillation (Protocol) 
TABLE OF CONTENTS

HEADER 1

ABSTRACT

BACKGROUND

OBJECTIVES

METHODS

ACKNOWLEDGEMENTS

REFERENCES

APPENDICES

HISTORY

CONTRIBUTIONS OF AUTHORS

DECLARATIONS OF INTEREST

SOURCES OF SUPPORT 
[Intervention Protocol]

\section{Oral Class I and III antiarrhythmic drugs for maintaining sinus rhythm after catheter ablation of atrial fibrillation}

Mazhar Warraich¹, Christina Peter², Mahmood Ahmad³, Shazaib Sheikh4, George R Abraham5, Muhammad Salman Sahibzada6, Ranu Baral7 $^{7}$, Adnan Muzaffar 8 , Joseph Tomson 5 , Pier Lambiase ${ }^{9}$, Gabriella Captur ${ }^{10,11}$, Amitava Banerjee ${ }^{12}$, Rui Providencia13

1Department of Internal Medicine, The Royal Wolverhampton Hospitals NHS Trust, Wolverhampton, UK. 2Department of Cardiology, Addenbrookes Hospital (Cambridge University Hospitals), Cambridge, UK. ${ }^{3}$ Cardiology Department, Royal Free Hospital, Royal Free London NHS Foundation Trust, London, UK. 4Department of Anaesthesia, St Helier Hospital, London, UK. ${ }^{5}$ Department of Cardiology, Royal Free Hospital, London, UK. ${ }^{6}$ Calderdale Royal Hospital, Halifax, UK. ${ }^{7}$ University of East Anglia, Norwich, UK. ${ }^{8}$ Scunthrope General Hospital, Scunthorpe, UK. ${ }^{9}$ Centre for Cardiology in the Young, The Heart Hospital, University College London Hospitals, London, UK. ${ }^{10}$ Royal Free Hospital, London, UK. 11MRC Unit of Lifelong Health and Ageing, University College London, London, UK. 12Institute of Health Informatics Research, University College London, London, UK. ${ }^{13}$ Barts Heart Centre, St Bartholomew's Hospital, Barts Health NHS Trust, London, UK

Contact address: Mahmood Ahmad, mahmood.ahmad2@nhs.net.

Editorial group: Cochrane Heart Group.

Publication status and date: New, published in Issue 10, 2020.

Citation: Warraich M, Peter C, Ahmad M, Sheikh S, Abraham GR, Sahibzada MS, Baral R, Muzaffar A, Tomson J, Lambiase P, Captur G, Banerjee A, Providencia R. Oral Class I and III antiarrhythmic drugs for maintaining sinus rhythm after catheter ablation of atrial fibrillation (Protocol). Cochrane Database of Systematic Reviews 2020, Issue 10. Art. No.: CD013765. DOI: 10.1002/14651858.CD013765.

Copyright $(2020$ The Cochrane Collaboration. Published by John Wiley \& Sons, Ltd.

\section{A B S T R A C T}

\section{Objectives}

This is a protocol for a Cochrane Review (intervention). The objectives are as follows:

To assess the effects of oral Class I and III antiarrhythmic drugs for maintaining sinus rhythm in patients undergoing catheter ablation, compared to catheter ablation alone, for atrial fibrillation (AF). 


\section{B A C K G R O U N D}

\section{Description of the condition}

Atrial fibrillation (AF) is the most commonly found abnormal heart rhythm condition. It is associated with increased morbidity and mortality through stroke caused by a blood clot and subsequent failure of the left lower chamber (ventricle) of the heart (Markides 2003; Poon 2005). Recent UK data have demonstrated an increase in the prevalence of AF by $50 \%$ from 2000 to 2016 , meaning the condition now affects $3.3 \%$ of the general practice population who are aged 35 years or older (Adderley 2019). The worldwide prevalence of $0.5 \%$ ( 33.5 million individuals) is likely to be an underestimation, given the large proportion of asymptomatic and undiagnosed individuals (Patel 2018).

Structural heart disease - both inherited and secondary to hypertension, ischaemia or valvular heart disease, hyperthyroidism, high body mass index (BMI), and many others - is a risk factor for AF, whilst AF without risk factors is only seen in $15 \%$ of AF cases (Markides 2003). Typically the abnormal electrical activity of AF arises from the muscle layer of the lung veins into the left upper chamber (atrium) of the heart (Markides 2003). Patients presenting with new-onset AF can be treated with rate control with or without anticoagulation, depending on their risk for stroke (Kirchhof 2017). Rate control, through the use of beta blockers, calcium channel blockers or digoxin, can be appropriate for patients with hypertension, structural heart disease and permanent AF. However, in some patients the AF needs to be eliminated and the normal heart rate (sinus rhythm) needs to be restored as they have severe symptoms or do not benefit from rate control therapy or prefer to treat their AF (Boriani 2018). Atrial tachyarrhythmias (ATa), including AF, atrial flutter or atrial tachycardias, can occur after ablation. In atrial flutter an electrical signal moves in a circular motion around the atrium, causing the atria to beat much faster than the ventricles. Atrial tachycardias originate from an ectopic focus in the atria (Kirchhof 2017).

\section{Description of the intervention}

Rhythm control in AF is achieved by antiarrhythmic drugs, ablation therapy or electrical cardioversion (Kirchhof 2017). Ablation therapy has emerged as an alternative in symptomatic or drugresistant patients and it is well established that catheter ablation is superior to oral antiarrhythmic drugs alone for rhythm control (Haegeli 2014). Catheter ablation uses a catheter to identify the abnormal electrical triggers causing AF, which are then neutralised using radiofrequency impulses or other methods (Haegeli 2014). Patients who are eligible for catheter ablation include those who are symptomatic or those intolerant to oral antiarrhythmic medication (either paroxysmal which is a class I indication or persistent which is a class Ila indication) (Calkins 2017). The 2016 European Society of Cardiology (ESC) AF guidelines, based on level A evidence (which means a strong recommendation for clinicians), recommend catheter ablation of symptomatic paroxysmal AF to improve AF symptoms in patients with symptomatic recurrences on antiarrhythmic therapy and who prefer further rhythm control therapy (class I indication) (Burns 2011; Kirchhof 2017).

Antiarrhythmic drugs which come in number of different types are used to maintain patients in sinus rhythm. The Vaughan-Williams classification of antiarrhythmics divides electrophysiological action of drugs by their effect on ion channels, as follows.
1. Class I agents interfere with the sodium $\left(\mathrm{Na}^{+}\right)$channel.

2. Class II agents are anti-sympathetic agents.

3. Class III agents affect potassium $\left(\mathrm{K}^{+}\right)$efflux.

4. Class IV agents affect calcium channels and the atrioventricular (AV) node.

5. Class $V$ agents work by other or unknown mechanisms.

The action of oral Class I and Class III agents on sodium channels and potassium channels, respectively, slows the repolarisation of cardiac myocytes and increases the refractory period; hence, these drugs are effective in rhythm control (Lei 2018). The other classes of drugs are often used in rate control and are less relevant for this review.

\section{How the intervention might work}

Prolonged AF causes structural and physiological changes that enable AF to continue. Failure of maintaining normal rhythm after ablation is not uncommon and thus post-procedural antiarrhythmic drugs (generally Class I and III drugs) have been thought to prevent recurrence of AF when used after ablation. Patients with early relapses in the 'blanking period' (a period of approximately three months, in which occurrences of ATa including AF, atrial flutter or atrial tachycardias - after ablation are disregarded) are more likely to relapse. These ATa are rhythms that originate from the atrium as a consequence of abnormal electrical activity and represent a failure of the ablation treatment. Patients can become relapse-free after the inflammation from the ablation subsides (Willems 2016). Antiarrhythmic drugs may play a role in reducing early relapses in the 'blanking period' during electrophysiological reorganisation, and thus reduce long-term relapse-producing ATa post-ablation. Class I and III antiarrhythmic agents can be well tolerated, however side effects are a serious concern (Markides 2003). Amiodarone in particular causes severe toxicity of the lungs, liver and eyes, among many other adverse effects. Class I drugs are pro-arrhythmic and can cause toxicity in multiple body systems (UI Reham 2015).

\section{Why it is important to do this review}

Although a previous meta-analysis by $\mathrm{Xu}$ and colleagues in 2015 concluded that antiarrhythmic drugs reduce the early reoccurrence of AF after catheter ablation, the researchers did not perform a detailed subgroup analysis ( $X u$ 2015). Early reoccurrence was found to be twice as likely without the use of antiarrhythmics; however, there was no significant difference in late reoccurrence (Xu 2015). It is, however, still thought that combination therapy (ablation and drugs together) is likely to be more effective than ablation alone. Multiple new trials have since been conducted and a new review is now required (Kaitani 2016; Duytschaever 2018). A recently updated Cochrane Review focused on the use of antiarrhythmic drugs after electrical cardioversion and concluded that the long-term benefit of antiarrhythmic drugs is unclear (Valembois 2019), however they may play an important role after ablation. Current guidelines from the National Institute for Health and Care Excellence (NICE) emphasise the need for expert opinion to guide the decision for ablation, however there is no guidance on the use of antiarrythmic drugs with ablation (NICE 2019). The American Heart Association (AHA) have agreed that the use of ablation is reasonable in symptomatic $A F$ in patients with heart failure, in order to lower mortality rate and hospitalisation (January 2019). Given the high cost and risk of complications of a repeat ablation, and the 
toxicity and dangers of antiarrhythmic drugs, this is an important question to answer. The latest ESC guidelines discuss the need for data comparing different antiarrhythmic interventions in patients with recurrent AF after catheter ablation (Kirchhof 2017).

\section{O B JECTIVES}

To assess the effects of oral Class I and III antiarrhythmic drugs for maintaining sinus rhythm in patients undergoing catheter ablation, compared to catheter ablation alone, for atrial fibrillation (AF).

\section{METHODS}

\section{Criteria for considering studies for this review}

\section{Types of studies}

We will include all published, unpublished and ongoing randomised controlled trials (RCTs) that are randomised at the level of the participant. We will not include cluster-RCTs (as this method of randomisation will introduce dependence and thus require further analysis), cross-over trials (due to the short followup period and the long-term effect of the drugs being used) or quasi-randomised studies (due to the risk of selection bias).

\section{Types of participants}

We will include adult participants (aged 18 years or older) of either sex who have had AF of any type or duration and had restoration of sinus rhythm with catheter ablation. For mixed populations, if only a subset meets the inclusion criteria, we will contact the trial authors to obtain subgroup data. If we cannot obtain the data for the subpopulation of interest, we will only include the study if a minimum of $60 \%$ of the study population meet the inclusion criteria (and we will explore the impact of this decision in the sensitivity analysis).

\section{Types of interventions}

1. Single Class I antiarrhythmics (flecainide, propafenone) versus control

2. Single Class III antiarrhythmics (amiodarone, dofetilide, dronedarone and sotalol) versus control

3. Combinations of any Class I and/or Class III antiarrhythmics versus control

Control is defined as standard medical therapy post-ablation alone and not including Class I or III antiarrhythmics or placebo or alternatively control also includes placebo and standard medical therapy post-ablation alone not including Class I or III antiarrhythmics. Medications other than Class I or Class III antiarrhythmics will be eligible as concomitant medications, provided they apply to all treatment arms.

\section{Types of outcome measures}

We will report outcomes from eligible studies that are included in our review. Reporting one or more of the outcomes listed below is not an inclusion criterion for a trial to be included in the review. Where a published report does not appear to report one of these outcomes, we will access the trial protocol and contact the trial authors to ascertain whether the outcomes were measured but not reported. Relevant trials which measured these outcomes but did not report the data, or not report them in a usable format, will be included in the review as part of the narrative.
As results from more than one time point cannot be combined without a unit of analysis error, we will present them at several periods of follow-up (zero to three months, greater than three months to six months, and greater than six months). If the followup period is unclear then we will use the longest follow-up data provided and narratively report this. Our main follow-up period of interest is greater than three months to six months, due to three months being considered a 'blanking period' where ATa are not uncommon as the body is recovering from the ablation.

\section{Primary outcomes}

1. Patients with recurrence of any ATa (AF, atrial flutter or atrial tachycardia) lasting greater than 30 seconds

\section{Secondary outcomes}

1. All-cause mortality

2. The number of participants who required at least one or more repeat ablations

3. Adverse events, considered separately as the following individual outcomes:

a. participants who have a thromboembolic event (including transient ischaemic attack, ischaemic stroke, deep vein thrombosis, pulmonary embolism and splanchnic vein thrombosis);

b. participants who have a myocardial infarction;

c. participants with a new diagnosis of heart failure;

d. participants who required hospitalisation one or more times for AF.

\section{Search methods for identification of studies}

\section{Electronic searches}

We will identify trials through systematic searches of the following bibliographic databases.

1. Cochrane Central Register of Controlled Trials (CENTRAL) in the Cochrane Library

2. MEDLINE (Ovid, from 1946 onwards)

3. Embase (Ovid, from 1980 onwards)

4. Web of Science Core Collection

The preliminary search strategy for MEDLINE (Ovid) (Appendix 1) will be adapted for use in the other databases. The Cochrane sensitivity and precision maximising RCT filter (Lefebvre 2011) will be applied to MEDLINE (Ovid) and adaptations of it to the other databases, except CENTRAL. We will also conduct a search of ClinicalTrials.gov (www.ClinicalTrials.gov) and the WHO International Clinical Trials Registry Platform (ICTRP) (apps.who.int/trialsearch) for ongoing or unpublished trials.

We will search all databases from their inception to the present, and we will impose no restrictions on language of publication or publication status. We will not perform a separate search for adverse effects of interventions used for the treatment of AF (i.e. with Class I and III antiarrhythmics). We will consider adverse effects described in included studies only.

\section{Searching other resources}

We will check reference lists of all included studies and any relevant systematic reviews identified for additional references to trials. We 
will also examine any relevant retraction statements and errata for included studies. Study authors will be contacted for missing data and ongoing trials as required.

\section{Data collection and analysis}

\section{Selection of studies}

Two review authors (MW and MA) will independently screen abstracts against the inclusion criteria and will classify them as eligible and not eligible. We will use Covidence software for this process. After retrieving the full-text papers, two review authors (MW and MA) will independently screen the full texts and identify studies for inclusion and exclude ineligible studies. Any disagreements at either stage will be resolved through involvement of a third review author (SS). We will identify and exclude duplicates and collate multiple reports of the same study so that each study, rather than each report, is the unit of interest in the review. The reasons for excluding full-text reports will be recorded and reported. We will complete a PRISMA flow diagram and 'Characteristics of excluded studies' table (Liberati 2009).

\section{Data extraction and management}

We will use a data collection form which has been piloted on at least one study in the review. Two review authors (MW, SS) will extract the following study characteristics from included studies.

1. Methods: study design, total duration of study, number of study centres and location, study setting, and date of study.

2. Participants: number $(\mathrm{N})$ randomised, $\mathrm{N}$ lost to follow-up/ withdrawn, $\mathrm{N}$ analysed, mean age, age range, gender, inclusion criteria, and exclusion criteria. In addition, we will collect data on left atrial size, percentage of paroxysmal and persistent AF and presence of comorbidities (mentioned in the background section).

3. Interventions: intervention, comparison, number of ablations per participant, concomitant medications, and excluded medications.

4. Outcomes: primary and secondary outcomes specified and collected, and time points reported.

5. Notes: funding for trial, and notable conflicts of interest of trial authors.

Two review authors (MW, SS) will independently extract outcome data from included studies. We will resolve disagreements by consensus or by involving a third person (MA). One review author (MSS) will transfer data into the Review Manager 5 file (Review Manager 2014). We will double-check that data are entered correctly by comparing the data presented in the systematic review with the data extraction form. A second review author (MA) will spot-check study characteristics for accuracy against the trial report.

\section{Assessment of risk of bias in included studies}

Two review authors (MA, MW) will independently assess risk of bias for each study using the Cochrane 'Risk of bias' tool, outlined in the Cochrane Handbook for Systematic Reviews of Interventions ( Higgins 2011) We will resolve any disagreements by discussion or by involving another author (SS). We will assess the risk of bias according to the following domains.

\section{Random sequence generation}
2. Allocation concealment
3. Blinding of participants and personnel
4. Blinding of outcome assessment
5. Incomplete outcome data
6. Selective outcome reporting
7. Other bias

We will judge each study to be at high, low, or unclear risk of bias for each of the domains listed, and we will provide direct quotes from the study report, with justifications for our judgement, in the 'Risk of bias' table. Where information on risk of bias relates to unpublished data or correspondence with trialists, we will note this in the 'Risk of bias' table. We are interested in quantifying the effect of assignment to the interventions at baseline, regardless of wether the interventions are received as intended (the 'intentionto-treat effect'). When considering treatment effects, we will take into account the risk of bias for the studies that contribute to that outcome.

\section{Measures of treatment effect}

The data we will collect will be dichotomous data and will be analysed as odds ratios with $95 \%$ confidence intervals.

\section{Unit of analysis issues}

If we identify any multi-arm trials, we will use the data from arms using the required approach (e.g. individual drugs versus placebo or no treatment) and exclude arms that are irrelevant to the scope of our review. For studies that include placebo and no treatment as two different controls, we will combine the control arms.

For studies that report more than one follow-up, outcomes at the longest possible time of follow-up will be analysed if the time point is not pre-specified (according to our primary outcome and first secondary outcome time point). We plan to analyse the different time points as separate comparisons to avoid a unit of analysis error.

\section{Dealing with missing data}

We will contact investigators or study sponsors in order to verify key study characteristics and obtain missing numerical outcome data where possible (e.g. when a study is identified as abstract only). Where this is not possible, and the missing data are thought to introduce serious bias, we will assume the data are missing at random and explore the impact of excluding such studies in the overall assessment of results by a sensitivity analysis (see Sensitivity analysis).

\section{Assessment of heterogeneity}

We will inspect forest plots visually to consider the direction and magnitude of effects and the degree of overlap between confidence intervals. We will use the $\mathrm{I}^{2}$, and $\mathrm{Tau}^{2}$ statistics to measure heterogeneity among the trials in each analysis. We acknowledge that there is substantial uncertainty in the value of $I^{2}$ when there is only a small number of studies. We will also consider the $P$ value from the $\mathrm{Chi}^{2}$ test.

If we identify substantial heterogeneity we will report it and explore possible causes by prespecified subgroup analysis. We will consider heterogeneity as substantial if there is a low $P$ value (less than 0.1) in the $\mathrm{Chi}^{2}$ test for heterogeneity, or if $\mathrm{Tau}^{2}$ is greater than 
zero. Strict thresholds for interpreting $\mathrm{I}^{2}$ are not recommended and we will therefore follow the rough guide as outlined in the Cochrane Handbook for Systematic Reviews of Interventions (Higgins 2017), where $50 \%$ or more represents substantial to considerable heterogeneity.

\section{Assessment of reporting biases}

If we are able to pool 10 or more studies, we will create and examine a funnel plot to explore possible small-study biases for the primary outcomes. In this case we will also perform a formal statistical test for asymmetry (e.g. Egger 1997). If there is only a small number of included studies then the ability to detect publication bias is largely diminished, so it will be difficult to exclude the presence of publication bias.

\section{Data synthesis}

We will undertake meta-analyses only where this is meaningful i.e. if the treatments, participants and the underlying clinical question are similar enough for pooling to make sense. We will carry out statistical analysis using Review Manager 5 software (Review Manager 2014). Due to clinical heterogeneity across trials with AF ablation, differences in comorbidities, differences in medication dose and additions of cointerventions, we will use a randomeffects model for our meta-analysis. We will look at the randomeffects summary as the average range of possible treatment effects. The average treatment effect will be given with a 95\% confidence interval. All eligible studies will be included regardless of their risk of bias; the outcomes will be assessed for risk of bias in the sensitivity analysis.

\section{Subgroup analysis and investigation of heterogeneity}

We plan to carry out subgroup analyses for the following factors for all outcomes but only for our primary time point of interest (greater than three months to six months).

1. Participants undergoing their first ablation versus those who have undergone successive ablations (to explore whether drugs are more or less successful in patients having undergone previous unsuccessful ablations). This subgroup analysis will be applied to all three planned comparisons.

2. Each individual drug (flecainide, propafenone, amiodarone, dofetilide, dronedarone and sotalol) versus control. This subgroup analysis will only be applied to comparison 1 and 2 under Types of interventions.

We will use the formal test for subgroup differences in Review Manager 5 (Review Manager 2014), and base our interpretation on this.

\section{Sensitivity analysis}

We plan to carry out the following sensitivity analyses on all outcomes measured at greater than three months to six months.
1. Only including studies with low overall risk of bias (we will consider a study to be at low risk of bias if it meets the criteria for low risk of bias in the following domains: random sequence generation, allocation concealment, and incomplete outcome data).

2. Only including studies without missing data.

\section{Summary of findings and assessment of the certainty of the evidence}

We will create separate 'Summary of findings' tables for each of the following three comparisons.

1. Single Class I antiarrhythmics (flecainide, propafenone) versus control

2. Single Class III antiarrhythmics (amiodarone, dofetilide, dronedarone and sotalol) versus control

3. Combinations of any Class I and/or Class III antiarrhythmics versus control

The 'Summary of findings' tables will include the outcomes listed under Types of outcome measures. Our 'Summary of findings' tables will cover all outcomes for the period of follow-up of greater than three months to six months.

We will use the five GRADE considerations (study limitations, consistency of effect, imprecision, indirectness and publication bias) to assess the certainty of the body of evidence as it relates to the studies which contribute data to the metaanalyses for the prespecified outcomes. We will use methods and recommendations described in Chapter 12 of the Cochrane Handbook for Systematic Reviews of Interventions (Schünemann 2017), using GRADEpro GDT software (GRADEpro GDT 2015). The overall 'Risk of bias' judgement for each study will be used as part of GRADE assessment. We will justify all decisions to downgrade the quality of the evidence using footnotes, and will add comments to aid the reader's understanding of the review if necessary.

Judgements about the quality of the evidence will be made by two review authors (MA, MW) working independently, with disagreements resolved by discussion or involving a third author (SS). Judgements will be justified, documented and incorporated into the reporting of results for each outcome. We plan to extract study data, format our comparisons in data tables and prepare a 'Summary of findings' table before writing the results and conclusions of our review.

\section{ACKN OWLEDGEMEN TS}

We are grateful to Cochrane Heart for providing the template for the protocol. We are grateful for peer reviewer Gh Andrei Dan, consumer reviewer Derek Ansel and the Cochrane Heart Editors for their input on the protocol. 


\section{R E F E R E N C E S}

\section{Additional references}

\section{Adderley 2019}

Adderley NJ, Ryan R, Nirantharakumar K, Marshall T. Prevalence and treatment of atrial fibrillation in UK general practice from 2000 to 2016. Heart 2019;105:27-33.

\section{Boriani 2018}

Boriani G, Proietti M. Atrial fibrillation prevention: an appraisal of current evidence. Heart 2018;104:882-7.

\section{Burns 2011}

Burns PB, Rohrich RJ, Chung KC. The levels of evidence and their role in evidence-based medicine. Plastic Reconstructive Surgery 2011;128(1):305-10. [DOI: 10.1097/ PRS.0b013e318219c171]

\section{Calkins 2017}

Calkins H, Hindricks G, Cappato G, Kim YN, Saad E, Aguinaga L, et al. HRS/EHRA/ECAS/APHRS/SOLAECE expert consensus statement on catheter and surgical ablation of atrial fibrillation: executive summary. Journal of Arrhythmia 2017;33(5):369-409. [DOI: 10.1016/j.joa.2017.08.001]

\section{Covidence [Computer program]}

Veritas Health Innovation Covidence. Version accessed 20 October 2020. Melbourne, Australia: Veritas Health Innovation. Available at covidence.org.

\section{Duytschaever 2018}

Duytschaever M, Demolder A, Phlips T, Sarkozy A, El Haddad M, Taghji P, et al. PulmOnary vein isolation With vs. without continued antiarrhythmic Drug trEatment in subjects with Recurrent Atrial Fibrillation (POWDER AF): results from a multicentre randomized trial. European Heart Journal 2018;39(16):1429-37.

\section{Egger 1997}

Egger M, Smith GD, Schneider M, Christoph M. Bias in metaanalysis detected by a simple, graphical test. BMJ 1997;315:629.

\section{GRADEpro GDT 2015 [Computer program]}

McMaster University (developed by Evidence Prime) GRADEpro GDT. Hamilton (ON): McMaster University (developed by Evidence Prime), 2015. Available at gradepro.org.

\section{Haegeli 2014}

Haegeli L, Calkins H. Catheter ablation of atrial fibrillation: an update. European Heart Journal 21 September 2014;35(36):2454-9. [DOI: https://doi.org/10.1093/eurheartj/ ehu291]

\section{Higgins 2011}

Higgins JP, Altman DG, Sterne JA (editors). Chapter 8: Assessing risk of bias in included studies. In: Higgins JP, Green S (editors). Cochrane Handbook for Systematic Reviews of Interventions Version 5.1.0 (updated March 2011). The Cochrane Collaboration, 2011. Available from www.training.cochrane.org/ handbook.

\section{Higgins 2017}

Higgins JP, Altman DG, Sterne JAC (editors). Chapter 8: Assessing risk of bias in included studies. In: Higgins JP, Churchill R, Chandler J, Cumpston MS (editors). Cochrane Handbook for Systematic Reviews of Interventions version 5.2.0 (updated June 2017). Available from www.training.cochrane.org/handbook.

\section{January 2019}

January CT, Wann LS, Calkins H, Chen LY, Cigarroa JE, Cleveland JC. 2019 AHA/ACC/HRS focused update of the 2014 guideline for management of patients with atrial fibrillation. Journal of the American College of Cardiology 2019;74(1):104-32.

\section{Kaitani 2016}

Kaitani K, Inoue K, Kobori A, Nakazawa Y, Ozawa T, Kurotobi T, et al. Efficacy of Antiarrhythmic drugs Short-Term use after catheter ablation for Atrial Fibrillation (EAST-AF) trial. European Heart Journal 2016;37(7):610-8.

\section{Kirchhof 2017}

Kirchhof P, Benussi S, Kotecha D, Ahlsson A, Atar D, Casadei B, et al. 2016 ESC guidelines for the management of atrial fibrillation developed in collaboration with EACTS. Revista Española de Cardiología 2017;70(1):50.

\section{Lefebvre 2011}

Lefebvre C, Manheimer E, Glanville J. Chapter 6: Searching for studies. In: Cochrane Handbook for Systematic Reviews of Interventions Version 5.1 (updated March 2011). The Cochrane Collaboration, 2011. In: Higgins JP, Green S, editors(s). Available from www.training.cochrane.org/handbook. The Cochrane Collaboration, March 2011.

\section{Lei 2018}

Lei M, Wu L, Terrar DA, Haung CL. Modernized classification of cardiac antiarrhythmic drugs. Circulation 2018;138(17):1879-96.

\section{Markides 2003}

Markides V, Schilling RJ. Atrial fibrillation: classification, pathophysiology, mechanisms and drug treatment. Heart 2003;89(8):939-43. [DOI: 10.1136/heart.89.8.939]

\section{NICE 2019}

Atrial Fibrillation. National Institute for Health and Care Excellence (NICE) 2019.

\section{Patel 2018}

Patel NJ, Atti V, Mitrani RD, Viles-Gonzalez JF, Goldberger JJ. Global rising trends of atrial fibrillation: a major public health concern. Heart 2018;104:1989-90.

\section{Poon 2005}

Poon K, Okin PM, Kligfield P. Diagnostic performance of a computer-based ECG rhythm algorithm. Journal of Electrocardiology 2005;38(3):235-8. 


\section{Review Manager 2014 [Computer program]}

The Cochrane Collaboration Review Manager 5 (RevMan 5). Version 5.3. Copenhagen: The Nordic Cochrane Centre: The Cochrane Collaboration, 2014.

\section{Schünemann 2017}

Schünemann HJ, Oxman AD, Vist GE, Higgins JPT, Deeks JJ, Glasziou P, et al. Chapter 12: Interpreting results and drawing conclusions. In: Higgins JPT, Churchill R, Chandler J, Cumpston MS (editors). Cochrane Handbook for Systematic Reviews of Interventions version 5.2.0 (updated June 2017). Available from www.training.cochrane.org/handbook.

\section{UI Reham 2015}

UI Rehman S, Siddiqui N, Khan NS, Sobia R, Assaly R. Multisystem side effects of amiodarone. American Journal of the Medical Sciences 2015;349(5):454.

\section{AP P E N D ICES}

\section{Appendix 1. Preliminary MEDLINE (Ovid) search strategy}

1 Atrial Fibrillation/ (52683)

2 atrial fibrillat*.tw. (65841)

3 atrium fibrillat*.tw. (13)

4 auricular fibrillat*.tw. (933)

51 or 2 or 3 or $4(77570)$

6 Flecainide/ (1679)

7 flecainide*.tw. (2186)

8 Propafenone/ (1340)

9 propafenone.tw. (1571)

10 Amiodarone/ (7523)

11 Amiodarone.tw. (9180)

12 Dofetilide.tw. (865)

13 Dronedarone/ (421)

14 Dronedarone.tw. (573)

15 Sotalol/ (2050)

16 Sotalol.tw. (2729)

176 or 7 or 8 or 9 or 10 or 11 or 12 or 13 or 14 or 15 or $16(16972)$

185 and 17 (3737)

19 randomized controlled trial.pt. (495635)

20 controlled clinical trial.pt. (93449)

21 randomized.ab. (462524)

\section{Valembois 2019}

Valembois L, Audureau E, Takeda A, Jarzebowski W, Belmin J, Lafuente-Lafuente $C$. Antiarrhythmics for maintaining sinus rhythm after cardioversion of atrial fibrillation. Cochrane Database of Systematic Reviews 2019, Issue 9. Art. No: CD005049. [DOI: 10.1002/14651858.CD005049.pub5]

\section{Willems 2016}

Willems S, Khairy P, Andrade JG, Hoffmann BA, Levesque S, Verma $A$, et al. Redefining the blanking period after catheter ablation for paroxysmal atrial fibrillation: insights from the ADVICE (Adenosine following pulmonary Vein Isolation to target dormant Conduction Elimination) trial. Circulation: Arrhythmia and Electrophysiology 2016;9:8.

\section{Xu 2015}

Xu X, Alida CT, Yu B. Administration of antiarrhythmic drugs to maintain sinus rhythm after catheter ablation for atrial fibrillation: a meta-analysis. Cardiovascular Therapeutics 2015;33(4):242-6. [DOI: 10.1111/1755-5922.12133]

Oral Class I and III antiarrhythmic drugs for maintaining sinus rhythm after catheter ablation of atrial fibrillation (Protocol) 
22 placebo.ab. (203209)

23 clinical trials as topic.sh. (189357)

24 randomly.ab. (322810)

25 trial.ti. (209005)

2619 or 20 or 21 or 22 or 23 or 24 or 25 (1254515)

27 exp animals/ not humans.sh. (4648880)

2826 not $27(1153980)$

2918 and $28(955)$

\section{H I S T OR Y}

Protocol first published: Issue 10, 2020

\section{CONTRIBUTIONS OF AUTHORS}

MA and MW wrote the first draft. CP, SS, MSS, PL, RP, AB, GA, AM, JT, RB and JC all edited and gave advice on the protocol.

\section{DECLARATIONS OF INTEREST}

MW: none known

CP: none known

MA: none known

SS: none known

GRA: none known

MSS: none known

RB: none known

AM: none known

JT: the author reports he or his department has received grants/sponsorship from Medtronic, Abbott, Boston Scientific, Microport and Bayer in the last three years. No grants or fees were received for this work. The author has also received speaker fees from Bayer (manufactures Xarelto).

PL: receives speaker fees and educational grant funding from Boston Scientific, and educational grant funding from Medtronic for work unrelated to the content of this Cochrane Review.

GC: none known

AB: I have been on advisory boards in work unrelated to the topic of this review with Pfizer, Astra-Zeneca, and Novo-Nordisk. A grant from Astra Zeneca was received by my institution. I am a trustee of the South Asian Health Foundation.

RP: none known

\section{SOURCES OF SUPPORT}

\section{Internal sources}

- Royal Free Hospital, UK

Contact author is employed as Cardiology Specialist Registrar at this hospital. 


\section{External sources}

\section{- NIHR, UK}

This project was supported by the National Institute for Health Research (NIHR), via Cochrane Infrastructure funding to Cochrane Heart. The views and opinions expressed therein are those of the authors and do not necessarily reflect those of the Systematic Reviews Programme, NIHR, NHS or the Department of Health and Social Care. 